\title{
ARTICULATION OF SPATIAL AND GEOMETRICAL KNOWLEDGE IN PROBLEM SOLVING WITH TECHNOLOGY AT PRIMARY SCHOOL
}

Sophie Soury-Lavergne

S2HEP

Institut Français de l'Education,

Ecole Normale Supérieure de Lyon, Lyon (FR)

sophie.soury-lavergne@ens-lyon.fr

\author{
Michela Maschietto \\ Dipartimento di Educazione e Scienze Umane \\ Università di Modena e Reggio Emilia, \\ Reggio Emilia (IT) \\ michela.maschietto@unimore.it
}

\begin{abstract}
Our paper focuses on the relationship between spatial and geometrical knowledge in problem solving situations at primary school. We have created tasks that involve three different spaces: physical space, graphical space and geometrical space. We aim to study the specific role of graphical space as a bridge between the other two spaces using paper and pencil and digital technology. We resort to the idea of dimensional deconstruction to design the tasks and to characterize the geometrical reasoning in pupils' problem solving processes. We organized a class-based intervention with 7year-old pupils to experiment a spatial problem involving the use of a grid and Cabri Elem e-book.
\end{abstract}

\section{INTRODUCTION}

The discussion about the teaching and learning of geometry has been going on in mathematics education research ever since the first studies that mathematicians and educators did at the turn of the twentieth century. This discussion is particularly relevant at the primary school level. Several theoretical approaches centered on the conceptualization of space (Piaget \& Inhelder 1963; Van Hiele 1986; Lurçat 1976) have been developed. Freudenthal wrote about "grasping space" as a function of geometry (Freundenthal, 1973, p. 477). Recent research in mathematics education focuses on the study of geometrical thinking through the identification of geometrical competencies at the secondary school level and also the relationships between them (Maschietto et al. 2013).

Despite this wide interest, there has been no consensus on what the teaching of geometry in primary schools should be. For instance, if we consider beginning learners at the kindergarten level, we can at least find two different perspectives. Within the frameworks of Van Hiele's levels, Fischbein's figural concepts and the duality concept image/ concept definition, Levenson et al. (2011) analyze preschool geometry in terms of children's conceptualization of geometrical figures. They focus on the construction of concept images and the distinction between critical/non-critical attributes of shapes, examples and non-examples [in this journal issue, Tsamir et al. (2015) analyze teachers' conceptualization of triangles, circles and cylinders].

Bryant (2009) considers several studies about children's understanding of their spatial environment based on everyday experience even before they attend school. He discusses geometrical learning in relation to the understanding of space. In particular, he refers to early spatial knowledge in his discussions, where perception plays an important role in recognizing shapes, size, orientation and position. Focusing on the same relationships between geometry and space, Clements and Samara 
(2009) take into account the development of spatial thinking and resort to particular physical and digital manipulatives as building blocks. These studies seem to emphasize a dominant role of spatial activities before the very start of teaching geometry at primary school and during the early years. However, Bryant (2009) claims that there is no strong evidence that this spatial understanding facilitates the development of geometrical thinking if we consider pupils' lasting difficulties in learning geometry.

Based on Bryant's (2009) work, the relationships between the learning of geometry and the understanding of space is an essential component of research work in this field.

\subsection{Spatial knowledge and geometrical knowledge}

In their research work on teaching geometry at primary school, Berthelot and Salin (1993) distinguish between spatial knowledge and geometrical knowledge and consider them as two different fields of knowledge. More precisely, spatial knowledge can be considered as a genuine field of knowledge, with its own problems, semiotic systems and operative invariants [in the sense of conceptual field, see Vergnaud (2009) and Balacheff (2013)]. It is not an informal form, or an embryo, or a subset of the more abstract and formal geometrical knowledge.

In the preface of his book "Elements of geometry", Clairaut (1741) explains how he resorts to spatial and field problems, and claims that it is the way geometers produce geometrical knowledge. He also warns the reader not to confuse his book with a treatise on land-surveying. Hence, his preface distinguishes the two fields of knowledge and points out their specific relationships. The main difference between spatial knowledge and geometrical knowledge is not the fact that one is less formal than the other, but the type of problems they investigate and the way their solutions are validated.

Geometry is a set of theoretical objects and relationships accessible by resorting to different semiotic systems, mainly graphical and linguistic. Validation is theoretical, independent from the subject and deals with the non-contradiction of geometrical properties. Regarding geometrical knowledge, objects and properties are expressed by the means of drawings and statements which require different kinds of approach and control by pupils (Parzysz, 1988). Statements call for a linear and an analytical approach under theoretical controls. Drawings call for a global perception, thus allow the visualization of spatial relationships and properties under perceptive controls. But drawings are not only a semiotic system to express geometrical concepts and reasoning. Drawings and graphics are also a means to model the perceivable surrounding environment (Laborde, 2004). Therefore, geometry is also a model of space and it is related to spatial knowledge.

Spatial knowledge addresses a different class of problems, such as characterizing shapes, positions and movements (creating a map, using a plan to achieve a construction or using a map to reach a destination). In the spatial field, validation is pragmatic and empirical. It results from a confrontation with the physical space and involves the subject and his/her perception.

Some vocabulary may be specific to one field of knowledge. For instance, horizontal and vertical directions, or above and below, are notions of the spatial field, because they are related to the impact of gravity on the surrounding environment. Studying the different kinds of language that a pupil uses 
may be useful to understand which knowledge s/he refers to. For instance, the use of words such as thin, thick, round, behind, in front of, indicates spatial references. But vocabulary alone cannot act as a specific characteristic of one field or the other. Some linguistic expressions, such as alignment or parallel, pertain to both references. Alignment of objects can be verified by eyesight; there is a position where the subject sees only one of the objects (when the objects are aligned with the subject). This makes alignment a notion of the spatial field. But alignment is also a notion of the geometrical field. Alignment concerns points and is defined by being on the same straight line, which is a theoretical object. Therefore, a single word isn't sufficient to specify spatial or geometrical field.

\subsection{Spatial knowledge in the learning of geometry}

Once the two fields of knowledge are recognized, questions regarding their relationship (Fig. 1) and how this relationship affects the learning of geometry are raised.

The development of geometrical knowledge provides a model of space and allows for the development of spatial skills: "Geometry lessons at school deal with the use of mathematics and logic to analyze spatial relations and the properties of shapes" (Bryant, 2009, p. 4). In return, spatial knowledge is a foundation for the construction of geometrical knowledge: "shape and space as fundamental ingredients for constructing a theory" (Hershkowitz, Parzysz, \& Van Dormolen, 1996, p. 162).

Following Berthelot and Salin's (1993) work, Perrin- Glorian et al. (2013) take into account pupils' difficulties in learning geometry. From their analysis of textbooks (projections of institutional expectations), they conclude that spatial knowledge is not sufficiently addressed in primary schools with respect to geometrical knowledge. In other words, teachers do not spend enough time undertaking spatial tasks with their pupils. From our point of view, the question is more complex than Perrin-Glorian et al.'s claims.

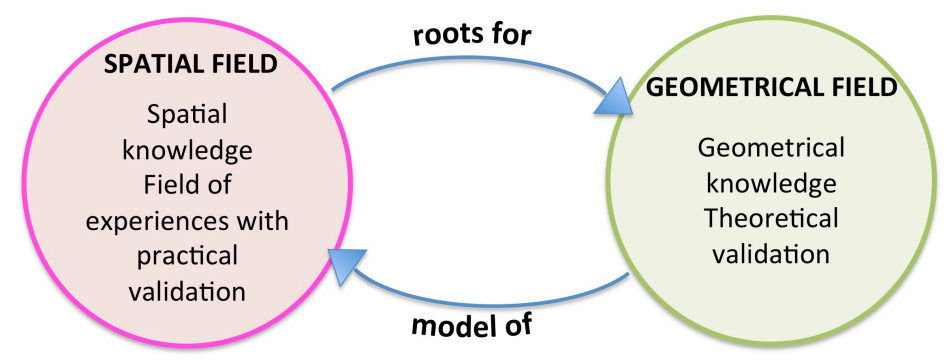

Fig. 1 Relationships between spatial field and geometrical field

If teaching geometry at primary school strives to give pupils tools to solve spatial problems beyond strategies strictly related to the context and the proposed situations, then the analysis of pupils' processes should not focus only on an adequate development of spatial knowledge in primary schools, but also focus on the development of relationships between spatial and geometrical knowledge in order to construct geometrical knowledge. From this perspective, this paper focuses on the study of relationships between spatial and geometrical knowledge. Our research lies in the identification of characteristics of situations likely to provide pupils with opportunities to build relations and to develop geometrical knowledge in order for pupils to handle spatial problems. In particular, we design 
learning situations based on the use of physical artifacts and digital technology in order to improve pupils' understanding of the relationships between the two kinds of knowledge, with the aim of rendering geometry a model of the spatial situations.

The paper is structured in two parts. After the elaboration of design principles derived from our theoretical study, we present a teaching experiment carried out at a primary school with 7-year-old Italian pupils.

\section{THREE SPACES IN INTERACTION IN THE LEARNING OF GEOMETRY}

Teaching geometry at primary school is difficult not only because teachers distrust their levels of expertise, but also because geometrical knowledge and geometry raise epistemological questions about the very nature of the type of knowledge to be taught. Solving geometry problems rely on two different fields of knowledge: the field of geometrical knowledge and that of spatial knowledge. Therefore, the question is on how to create relationships between the two fields of knowledge.

\subsection{The graphical space in the teaching and learning of geometry}

In recent years, Perrin-Glorian et al. (2013) have distinguished three kinds of spaces interacting in the teaching and learning of geometry: physical space, graphical space and geometrical space (Fig. 2).

According to them, physical space is the world of physical objects which pupils perceive and act on, that is, the "real" world where a concrete problem is to be solved. Geometrical space contains Euclidian theory, deductions and axioms, and provides pupils with tools to solve problems. Graphical space contains diagrams, drawings, schemas and artifacts which pupils use to solve problems. PerrinGlorian et al. (2013) pay particular attention to graphical space, which acts as a 'bridge' between the other two spaces [in this journal issue, Thom and McGarvey (2015) study how drawings, an element of the graphical space, contribute to pupils' geometrical understanding]. "The graphical space is a place to experiment to solve the problem arising in the physical world as well as the theoretical problem (it may play the role of interface between physical space and geometrical space)" (PerrinGlorian et al. 2013, p. 19). Indeed, two different views on the graphical space are possible. If a modeling problem of physical space is considered, the bi-dimensional graphical space contains diagrams or representations of objects. As soon as a representation is produced, a bridge from the tridimensional physical space [that can be macro, meso or micro for the subject (Berthelot \& Salin, 1993)] to a bi-dimensional micro graphical space (paper or screen) is formed. But if a theoretical problem is proposed, the graphical space contains figures representing theoretical objects.

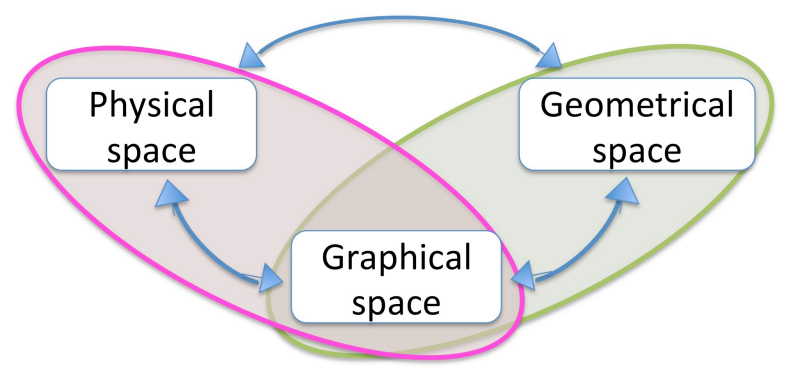

Fig. 2 Three interacting spaces in the learning of geometry 
Within the scope of this paper, we put forward the hypothesis that spatial knowledge is more useful and efficient to cope with problems situated in the physical and/ or graphical spaces, and geometrical knowledge is required only as a second resource when modeling appears to be a means to deal with the problem. Conversely, geometrical knowledge is more useful and efficient to cope with problems of the graphical and/or geometrical spaces, even if spatial knowledge may also be involved to build and explore representations of the problem.

In this paper, we focus on grids that are found in primary school textbooks and proposed in tasks concerning paths and position of objects. In this context, the grid is a graphical object in the micro graphical space that can be used to evoke meso space movements, and a geometrical tool that can be used to model and solve spatial tasks.

\subsection{Dimensional deconstruction, a new insight on geometrical activity}

According to Duval (2005), dimensional deconstruction of geometrical shapes is the central process of geometrical visualization. We introduce this notion because our epistemological considerations (spatial and geometrical duality) and the three spaces are not sufficient to provide criteria to recognize the subject's geometrical activity in the problem solving process.

The dimensional deconstruction of shapes is the mechanism by which one identifies several possible figural entities that constitute a given figure and their geometrical relationships. A figure, as a theoretical object, is represented by a diagram among a set of possible diagrams that belong to the graphical space (Perrin-Glorian et al. 2013). The dimension of each of these figural entities embedded in the figure may vary from 3 to 0 . For instance, a cube (if the inner part is included) is a threedimensional object; polygons, angles etc. are two-dimensional objects; lines, circumferences etc. are one-dimensional objects and points are zero-dimensional objects. Duval (2005) claims that the essence of geometrical activity when solving a geometrical problem is the deconstruction of the figure, where one considers entities of a smaller dimension than the initial figure and related properties. For instance, when working on a cube, one can consider it as a single global 3D entity, a set of faces (2D), a set of edges (1D), or a set of vertices (0D). When working with a square, considering the square as a set of edges is a $2 \mathrm{D}$ to $1 \mathrm{D}$ deconstruction, and considering it as a set of vertices is a $2 \mathrm{D}$ to $0 \mathrm{D}$ deconstruction. Mithalal (2010) studies pupils' strategies in the task of constructing a missing vertex in a cube. He shows that strategies that involved the construction of the missing tetrahedron (3D to 3D) or triangles (3D to $2 \mathrm{D}$ ) were preferred to strategies that involved the construction of the point symmetric to an existing point (3D to $0 \mathrm{D}$ ).

Duval (2005) claims that none of the tasks generally used at the primary school level helps pupils to develop cognitive processes of dimensional deconstruction of figures.

Our aim is to create geometrical situations and learning environments that require dimensional deconstruction, such as $3 \mathrm{D}$ to $2 \mathrm{D}$ or $2 \mathrm{D}$ to $1 \mathrm{D}$, so as to enable pupils to acquire progressively the process of geometrical reasoning. 


\subsection{Technology, an extended graphical space to bridge physical and geometrical spaces}

Dynamic geometry technology highlights the relationship between diagrams and figures. Dragging produces a continuous range of diagrams, which provides a way to deal with geometrical objects (Laborde \& Capponi, 1994).

We want to point out that a dynamic geometry environment also creates a link between the physical and graphical space as defined by Perrin-Glorian et al. (2013). Actions in dynamic geometry carry some of the properties of physical space, since objects can be moved, grabbed, suppressed, hidden, folded, etc. Dynamic geometry is also a graphical space, since one can produce different graphical representations of objects by referring either to the physical space or the geometrical space. Dynamic geometry provides pupils with continuous feedback as they drag the points of a diagram. This is something that cannot be produced using a paper and pencil diagram. In this sense, dynamic geometry can be considered as a graphical space with enlarged functionalities. Therefore, dynamic geometry could play a role in bridging the different spaces.

Beyond dynamic geometry, a key aspect of the added value of digital environment lies in feedback (Laborde et al. 2006). Indeed, feedback does exist in the physical space, but its nature is different from those provided by digital learning environments. We have identified three classes of feedback that apply to digital learning environments (Mackrell, Maschietto, \& Soury-Lavergne, 2013). Direct manipulation feedback includes every response of the environment to a user's actions, for instance, the continuous display of the positions of dragged points. Strategy feedback is a response of the system to the user's actions that are significant in the problem solving process. Finally, evaluation feedback is the system's assessment of the user's answer, and appears as a response to the user's demand (for example, the "check answer" button). Feedback creates key elements to highlight the interaction between the user and his/her environment. Feedback is also a major element in the use of digital technology enabling researchers to design learning environments different from the physical space. For instance, in the physical meso space the environment alone cannot always give relevant and useful feedback that enables the validation of strategies.

\section{QUESTIONS}

In the previous section, our epistemological study began with the distinction — and the relationshipsbetween the field of geometrical knowledge and the field of spatial knowledge. Our initial question focuses on how pupils construct relationships between the two fields of knowledge and how learning situations support this construction. We design and investigate situations that require pupils to work in the three spaces (physical, graphical and geometrical) and to develop relationships between them. They use digital technology and call for dimensional deconstructions.

Following Perrin-Glorian et al.'s (2013) work, we focus on the graphical elements and diagrams that are involved in learning situations. Pupils can consider them as models of spatial situations and possibly as representations of geometrical objects. We also take into account the graphical productions of the pupils. What kinds of pupils' actions, in terms of graphical elements in diagrams, can be used to attest the relationship between the spatial field and the geometrical field? 
Since the graphical space is not reduced to paper and pencil, digital environments allow pupils to produce and interact with graphical representations. Digital objects of the interface, along with paper and pencil diagrams, are possibly the model of a spatial situation and the representation of geometrical objects. Moreover, digital representations have properties that magnify those in paper and pencil because of the different kinds of feedback. Therefore, another feature of our proposal is to build situations involving a hybrid environment made up of two types of artifacts (a duo of artifacts in Maschietto \& Soury-Lavergne, 2013), one constituted by physical artifacts and another by digital technologies.

Our research question turns out to be so. Do learning situations that involve the use of manipulatives in the physical space the use of paper-and-pencil and digital technology in the graphical space help to envision a relationship between spatial knowledge and geometrical knowledge?

The last item of our proposal is to consider the work by Duval (2005). If geometrical reasoning involves some levels of dimensional deconstructions, we have to elaborate learning situations that require dimensional deconstructions and be able to recognize the occurrence of dimensional deconstruction in the pupils' discourse as evidence of their geometrical reasoning. More precisely, (1) which dimensional deconstructions, required as a way to solve the situation geometrically, can be observed in pupils' activities and in their graphical productions? (2) What are the critical aspects of such a learning situation that would support or prevent transfers between spatial and geometrical fields?

\section{METHODOLOGY BASED ON THE DESIGN OF TEACHING SCENARIOS INVOLVING DIGITAL TECHNOLOGY AND PHYSICAL SPACE}

A specificity of our research is a strong collaboration with teachers in the design and the implementation of teaching scenarios. Indeed, one of our aims is to provide teachers with innovative learning environments to improve everyday classroom practices. In order to achieve this goal, we conduct research "in the world of practitioners" and "develop empirically tested and theory-based solutions" (Stylianides \& Stylianides, 2013, p. 334). The learning situations we create have to implement our theoretical principles and to be compatible with the real contexts of classes and fulfill the requirements of the educational institution. This methodology shares some principles with the design-based research framework for educational research (Coob et al. 2003), which aims to solve complex problems in genuine context and in collaboration with teachers.

But classroom-based interventions, designed to experiment teaching and learning principles, generate constraints on the nature of the data we collect and analyze. For instance, while classroom-based interventions are well compatible with gathering pupils' interventions in collective discussion, they are not useful in recording every pupil's contribution during the entire scenario in the context of everyday classes. Hence, while the analysis can provide key elements of the class' reaction to the scenario, it requires another methodology to investigate individual pupil's learning trajectory. Nevertheless, the data provided by such experimental methodology are appropriate to decide if the principles, implemented in the experimental situations, are relevant to understand the relationships between spatial and geometrical knowledge. 


\subsection{Design principles}

Our teaching scenario allows us to experiment situations designed as a combination of the principles presented above: (1) the construction of relationships between spatial and geometrical knowledge, (2) the articulation of the physical, graphical and geometrical spaces by implementing elements of continuity between the spaces and (3) the use of digital technology to bridge them.

Moreover, the role of dimensional deconstruction is twofold. It is a design principle, like the examples mentioned previously. Our situations require partial dimensional deconstruction, certainly not a complete one going as far as $0 \mathrm{D}$ (when the construction of intersection points is required). Dimensional deconstruction is also a tool to analyze data. It is used as an indicator of pupils' geometrical reasoning.

The experiment (Sect. 5) focuses on the use of a grid to produce paths and to locate positions on a plane. The grid is an element present in primary school textbooks, in teaching practice and often in national evaluations. In teaching practice, tasks with grid are proposed within the graphical space, evoking spatial situations. The grid refers to the space in which a character can move. In this paper, we are interested in studying the relationships between the grid as a graphical object and the grid as a geometrical instrument to solve a spatial problem (Fig. 3). Hence, we mainly investigate geometry as a model to solve spatial problems (lower arrow in Fig. 3). The spatial problem used in this study requires participants to put down an object on a surface (a rectangular green carpet), remove it, and put it back to the original place. The key component of the experiment is the choice of spaces in which pupils are supposed to work: a micro graphical space and a meso physical space.

The dimensional deconstruction involved may be $2 \mathrm{D}$ to $1 \mathrm{D}$, because the grid can be considered as an assembly of squares (2D) or a net of intersecting lines (1D).

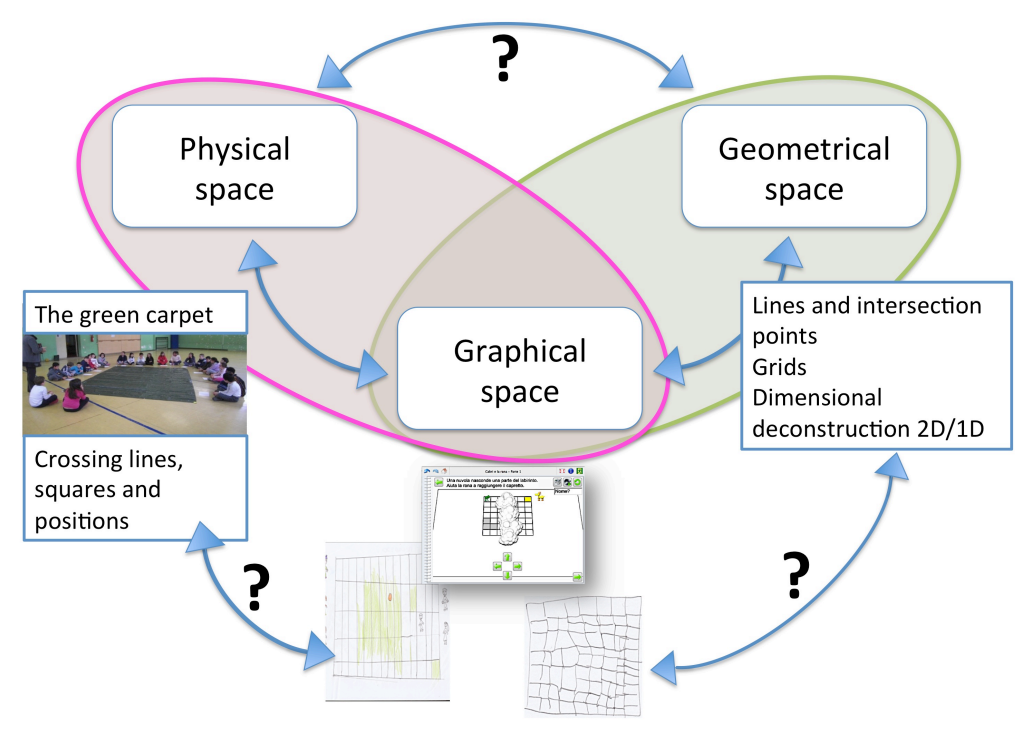

Fig. 1 The grid experiment 


\subsection{The Cabri elem technology and the e-book "Cabri and the frog"}

For our experiment, we need a kind of technology that offers the affordances of dynamic geometry, which relates to the physical space and generates feedback.

A wide range of digital technologies (including the use of robots, see Bartolini Bussi \& BaccagliniFranck, 2015, this journal issue) are available for dynamic diagrams and geometrical representations. There has been research involving the use of stand-alone applets and virtual manipulatives at the primary school level, as well as research involving DGEs such as Sketchpad (see Jackiw \& Sinclair, 2006, or Kaur, 2015, in this issue). However, we have chosen Cabri Elem because it has been specifically designed for use at the primary school level, with a range of functionalities going beyond what is typically found in DGEs.
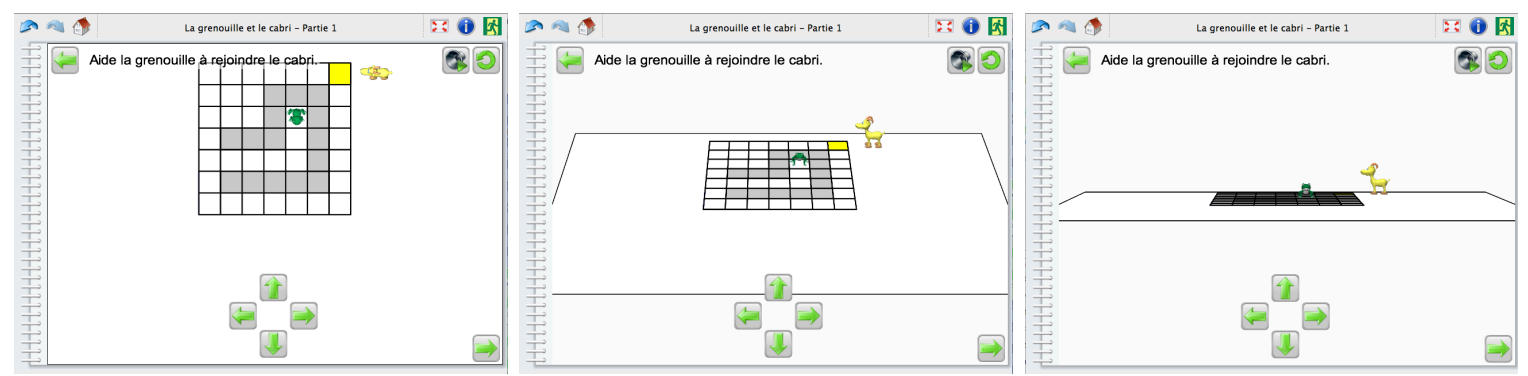

Fig. 2 The Cabri Elem user can continuously change the point of view on the object (direct manipulation feedback)

Cabri Elem takes on the richness of direct manipulation of Cabri geometry and includes some of the Cabri 3D functionalities such as a continuous transition between 2D and 3D points of view (Fig. 4). It also enables e-book designers to organize the tasks over a succession of pages. Designers can create representations of mathematical objects, include everyday life images or 3D models (such as animal images, Fig. 5), define the kind of action to be performed by the user (like moving or creating objects) and create feedback. Designers can implement a range of conditional feedbacks according to precise actions by the user, including direct manipulation feedback (like displaying or hiding objects, sounds), strategy feedback and evaluation feedback (Fig. 6).

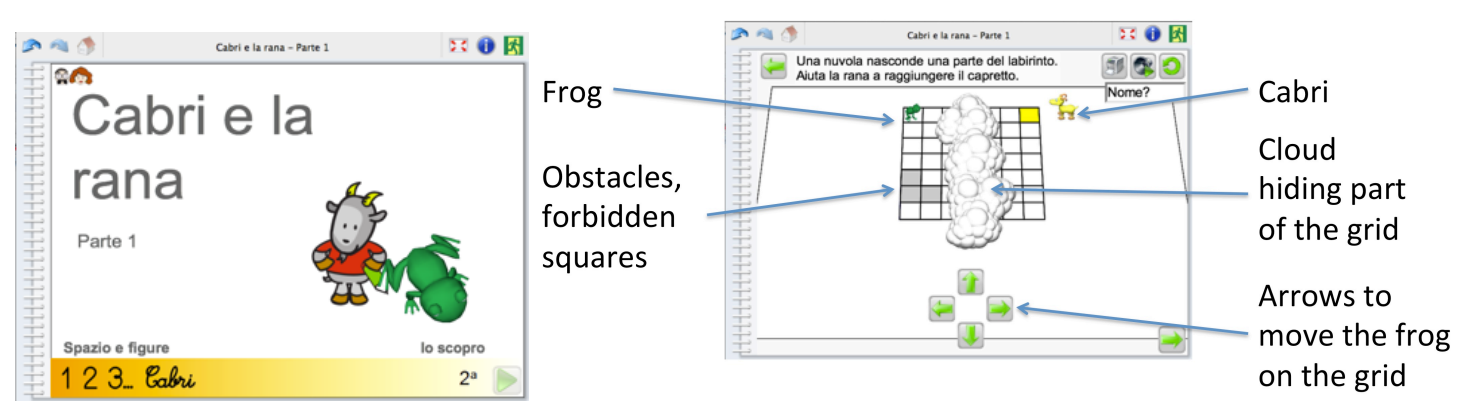

Fig. 3 Pages of the e-book "Cabri and the frog" 
The Cabri Elem e-book "Cabri and the frog" is made up of ten pages (Figs. 5, 6). Page 2 of the ebook introduces the task, to move the frog through the grid to reach Cabri, and suggests to use paper and pencil as tools to solve the task.

Each page displays: a grid with a frog and Cabri; four arrows for moving the frog; the task instruction "Help the frog to meet Cabri", written and audio instructions; a button to repeat the audio instruction and a button to reset the task. When an arrow-button is clicked, the frog moves on the grid according to the direction of the arrow. A continuous change in the points of view (passage 2D to 3D) is possible. From one page to another, obstacles, represented by grey squares and clouds hiding parts of the grid, come up. At first clouds flash for a certain time, then they remain static on the grid. On the last page, the clouds hide the grid all the time so as to spur pupils to rebuild the path. The configuration of the obstacles, the clouds and their behavior are didactical variables of the situation, inducing changes in pupils' problem solving strategies. For instance, the clouds covering the grid compel pupils to write down information about the path or the obstacles, engaging them in a strategy different from the visual control to reach the goal.

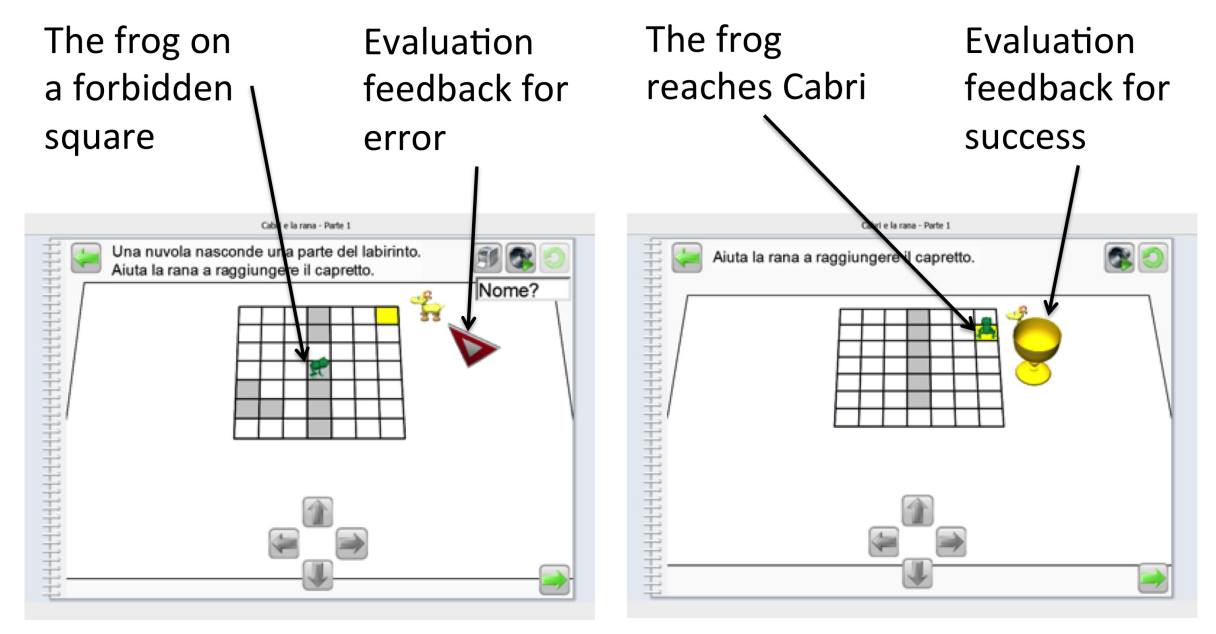

Fig. 4 Two pages of the e-book "Cabri and the frog"

The e-book provides some evaluation feedback to pupils. If the frog meets Cabri, a gold cup appears (Fig. 6, right). If the frog moves outside of the grid or on a forbidden square, a red triangle appears on the page (Fig. 6, left). The red triangle provides pupils with strategy feedback. Indeed, pupils can reload the activity and adjust the path, taking into account the information obtained from the previous trials. This allows pupils to build up on their problem solving strategy for the last page, where the cloud is never removed.

\subsection{Experimental setting}

In 2013, two teachers carried out the experiment with their classes of 7-year old pupils. They created the physical space activity with a green carpet as a complementary activity to the e-book "Cabri and the frog". 
Two classes are involved in the experiment. Class A (25 pupils, $13 \mathrm{~h}$ ) followed the structure of the three phases described in part 5 of this paper, while class B (21 pupils, $11 \mathrm{~h}$ ) went back to the green carpet after phase 3 .

The analysis is based on the videotapes of each lesson of class A (it was not possible to videotape class B), transcriptions of the collective discussions in the two classes made by the two teachers, and pupils' drawings (classes A and B).

Based on the data collected, we were able to analyze pupils' drawings, pupils' verbal interactions with the teacher and with other pupils during collective discussions and some pupils' problem solving strategies. Our observations focused on dimensional deconstruction and the connections pupils made between spatial and geometrical fields.

Dimensional deconstruction may be observed in pupils' diagrams when they represent 2D elements, like squares, rectangles, circles, in connection to (or not) to 1D elements like lines or segments and intersection points (0D elements). Dimensional deconstruction is also observed in pupils' verbal expressions, referring either to a $2 \mathrm{D}$ object (row) or to a $1 \mathrm{D}$ object (line).

Connections between spatial and geometrical field are seen in pupils' verbal expressions (near, in front of, on the line or intersection) and in their problem solving strategies (using the paper-pencil grid to solve a spatial problem).

\section{THE GRID EXPERIMENT}

The pupils had previously worked with path and grid on paper and pencil, but had never used Cabri Elem e-books prior to this study.

\subsection{The teaching plan and a priori analysis}

Phase 1 Problem solving in the meso physical space

The problem concerns the position of objects within the meso physical space. The whole unstructured 2D space is represented by a rectangular carpet laid on the floor. The teacher places an object on it, asks the pupils to take it out and then to relocate it at its original place. The pupils are invited to look for a strategy to solve the problem. Successful strategies may involve the use of additional elements to structure the plane. The problem solving process in the physical space may include the use of physical landmarks, including pupils themselves and other objects available in class. The use of a grid, or at least a reference to a grid, is expected to appear because it was introduced in previous activities. Of course, this requires pupils to create at first a link between the grid of the graphical space and the problem of the physical space. Pupils worked on the task several times, with the object located more and less distant from the edges of the carpet.

Phase 2 The grid on the carpet

With the assumption that the grid is one of the possible solutions proposed by the pupils, a second green carpet marked with a grid was prepared. Using this gridded carpet, the pupils may call on their knowledge constructed during their paper and pencil problem solving tasks in the micro-graphical space. It is relevant to analyze if and how the pupils use the graphical grid of their previous work as 
a model for the carpet in the meso space. Furthermore, the request of representing and communicating their strategy can facilitate the connection between the physical space and the graphical space. In their representations, geometrical knowledge may appear in terms of relation between the lines on the carpet, the intersections of those lines, and the relationship between the lines. Each of these properties requires some dimensional deconstruction and therefore indicates work in the geometrical space.

\section{Phase 3 The use of the technology}

Pupils worked in pairs in the micro graphical space of a computer screen with the e-book "Cabri and the frog". This e-book offers some continuity with the situation in the physical meso space. It uses graphical representations and increases the complexity of the task. The frog moves on the squares rather than along the lines, and pupils need to use the grid to solve the problem. As in the phase 2, the graphical space with paper-and-pencil can be used to support pupils' strategies. The e-book also provides pupils with strategy feedback, which is new in comparison to the tasks carried out previously using paper and pencil.

\subsection{Analysis of pupils' strategies in the physical space}

\section{Phase 1}

The duration of this phase differs in both classes. Class A spent two lessons on phase 1 of the experiment, while class B spent one lesson. After the pupils described the carpet, the teacher proposed the task described above.

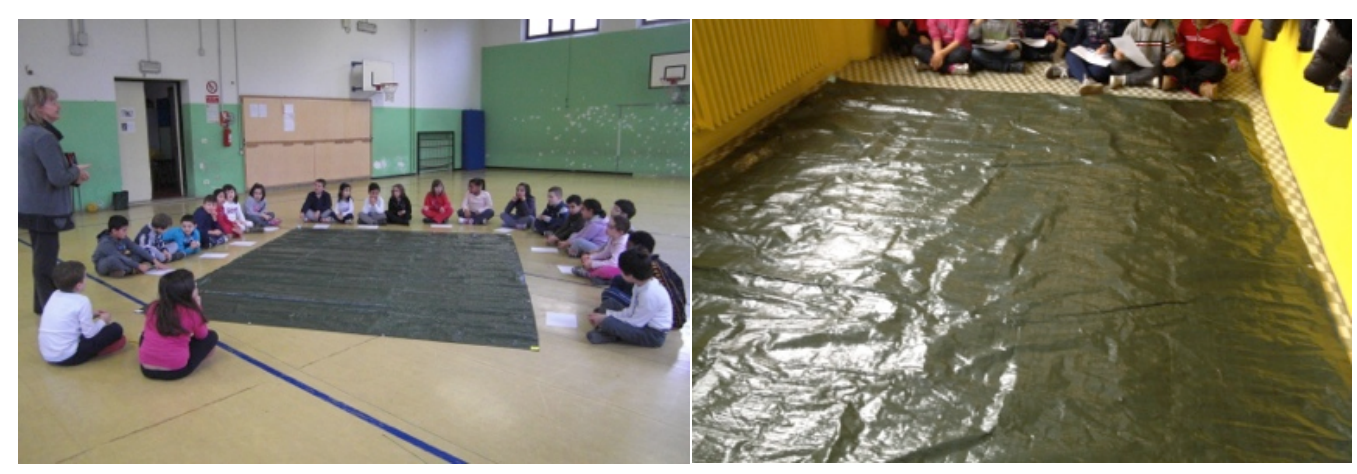

Fig 5 The carpets (classes A and B)

According to the teachers' report, pupils found the task easy when the object was located near an edge of the carpet (first attempt), but found the task difficult when the object was placed towards the center of the carpet. While the aim of the task was clear, they were not certain about the strategies they could use to solve the task.

In the two classes, several strategies appeared.

1. Strategies involving a visual-perceptive control and memory

This is coherent with the spatial situation where control involves perception and vision (class A, Samuele: "Look at the object very well", Matteo: "a bit more ahead"; class B, Alessandro: "but just 
remember where you took it"). This visual-perceptive control is also indicated by the proposition of adopting a point of view over the carpet (class A, Terry: "Look at from above"). These strategies are hardly abandoned even when inefficient (class A, lesson 3, Diletta: “... I have only used my memory").

\section{Strategies based on landmarks}

After the first attempt to solve the task, in each of the two classes, some pupils referred to the necessity of looking for "reference points". Landmarks were chosen outside and on the carpet, and the collective discussion made them explicit for the pupils. For internal landmarks, some pupils used creases or other particular features of the carpet (i.e., "a black line", "a mound", "a crease", see Figs. $7,8)$ that made it a non-homogeneous space. The pupils realized that the solution can be based on elements or people (external landmarks) that were present in the physical meso space in which they worked (e.g., in the gym or in the corridor, "before, it was closer to the radiator" (Mattia, class B); or on pupils sitting down around the carpet, "I remember that there was a child in front of the circle").

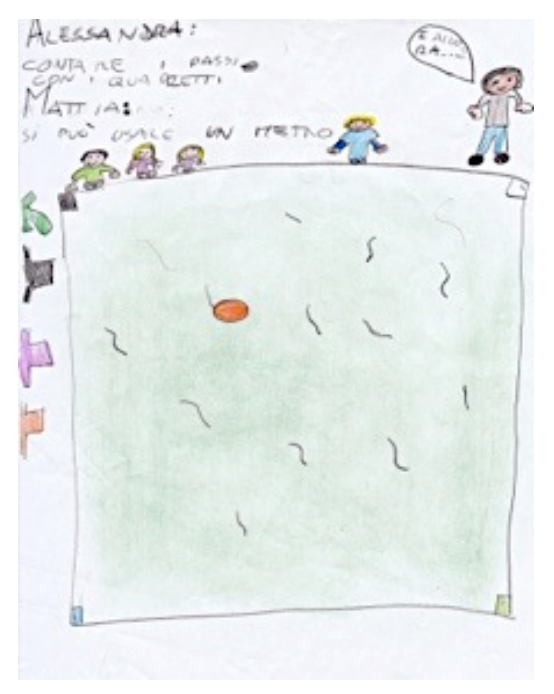

Fig 6 Drawing at the end of phase 1 (class B)

Pupils expressed the idea of lines and positions of reference operative in the meso physical space. The following is an excerpt of class A:

Matteo You [teacher] have placed an object, and then you have asked us to put it back in the right place; and we have done it using our schoolmates as a reference point.

Maya We have also used the cards on the vertices.

Matteo In fact, I have also used the cards for placing the object, and I also used an imaginary line to reach the center! The swelling of the carpet was another point of reference for me.

Teacher So what have you considered as a reference point?

Matteo A line, a line.

Teacher And you, Giulia, what did you use as reference point to relocate the object? 
Giulia I have looked at the vertices [of the carpet] and a line.

Teacher So you have also taken a line...

Giulia I have looked at the vertices and Alessandro P.

Teacher So you have referred to Alessandro P., and then you place the object?

$[\ldots]$

Matteo In fact, Alessandro has a long line in front of him!

The drawings collected at the end of phase 1 invoke the representation of the situation and the meso space in the micro graphical space ("making a plan", Fig. 8). They correspond to a 3D to 2D deconstruction.

The drawings reveal that the strategy of landmarks is based on the identification of lines or rows and positions on these lines, indicated by arrows (5/11 pairs of pupils of class A, Fig. 9), which are not elements of the physical space (not materialized on the carpet). These lines carry two purposes for the pupils: directions of movement on the physical space and straight lines of the geometrical space (2D to $1 \mathrm{D}$ deconstruction). In any case, these lines are $1 \mathrm{D}$ objects that do not intersect in a geometrical way, because the position is not represented by an intersection point but by a circle (or a bold dot which is placed in the center of the square, Fig. 9). The arrows that are perpendicular to each other or the lines pupils drew in the diagrams (Fig. 9) may indicate a primary structure of the space, even if they are strictly related to direction of motion starting from the sides of the carpet.
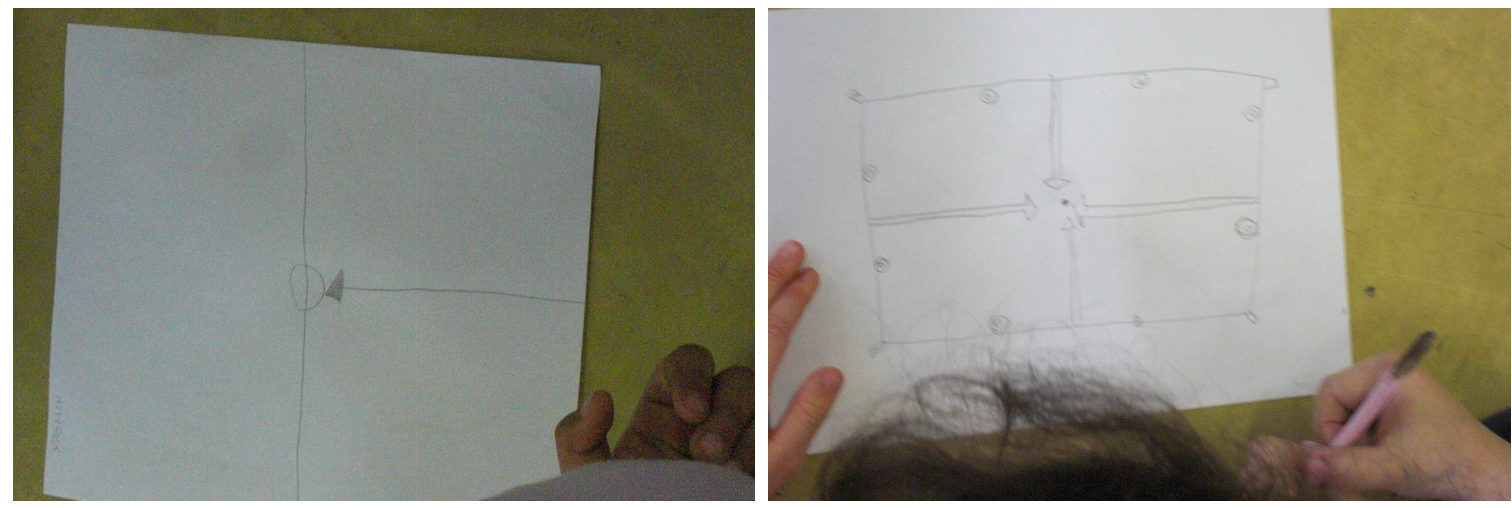

Fig 7 Drawings for the strategy of two lines, class A

\section{Strategies based on a grid}

During the discussion of class A, the pupils' remarks on how to use the lines and the way they considered the lines evoke the idea of grid that had been previously studied. However, pupils referred to the grid as an object that belongs to the micro graphical space. This can explain why the pupils did not refer to it in their problem solving strategies. In class B, only one child suggested the use of a grid with the expression "with small squares", which is an element of dimensional deconstruction:

Teacher We have to think very well, to look for a solution to this problem.

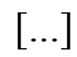


Alessandra With small squares another point of reference for me.

Teacher In your opinion, could the small squares help us?

Alessandro Yes, but if there are a lot of small squares, how do we remember on which squares you have put the object?

Unexpectedly, the grid did appear late in collective discussions and was not proposed as a means to structure the space and solve the task. But the teachers used pupils' interventions about squares and lines to introduce the second carpet. The following excerpt concerns the end of the discussion in class A (second lesson), in which the conception of the grid as an object, which belongs only to the micro graphical space appears:

Teacher And, what do several horizontal and vertical lines make?

Alessandro F. A grid!

Teacher And can we draw it?

Pupils [all together] Yes.

Teacher In your opinion, can we draw it on the carpet or on the paper?

Pupils On the paper!

\section{Strategies of measuring}

Pupils proposed to use a meter tape to measure the distance between the shape and a side of the carpet or to count steps to get to the shape. But the pupils failed to carry out this strategy [similar to Bryant's (2009) work]. The following is an excerpt of class B:

Teacher Children, we have seen that it was difficult to put the object in the exact point [position] where I had placed at the beginning. Thus, how can we overcome this difficulty? What could be the solution? How can we put the object in the exact position where it was?

Mattia We should have a meter to calculate.

Alessandra We can count the footsteps.

Teacher Do we try to follow Alessandra's suggestion? [the teacher invites Alessandra to solve the tasks by counting the footsteps]

Teacher Did Alessandra succeed in putting the shape in the right position?

Children [all together] Noooo!

Teacher Why didn't Alessandra's suggestion work?

Valentina First she walked four steps, when she returned, she walked fewer steps.

Emma First she made longer footsteps, then [she made] shorter [footsteps].

Alessandro I propose advice... It is true that counting footsteps can help us, but you might change the level [i.e., length] of the footsteps, before you make them short and then longer, then while she was going out the carpet, she has changed direction. Thus, you do not guess more, but if there is a particular element... For instance, a 
crease, you remember that there was a crease in that point, and you go there to put [the object].

The analysis of the first phase shows that the situation is meaningful, because the pupils were engaged in looking for a strategy. Their solutions used mathematical elements, such as measurements, lines and reference points, to manage the spatial situation. In the search for a strategy, some critical aspects come up concerning the language and the control of the solution. Pupils did not succeed in giving instructions to reproduce their path. Pupils around the carpet worked in the spatial space and used spatial language implicitly involving the choice of one reference ("it is closer to the center") or in relation to the position of the subject ("it was more down", "you put it to the right"). Pupils insufficiently alluded to spatial references to construct a robust structuration of the space, as in this excerpt (class B):

Alessandro Have you noticed that there's a black line here? [...] I have been watching, it was more down, next to the line.

Manuel In my opinion, it was not on the black line before. It was a bit more on the right.

\section{Phase 2}

In both classes, the teacher introduced the square carpet following some pupils' remarks in the collective discussions. With the same formulation, the task changed because of the implicit request to use the grid.

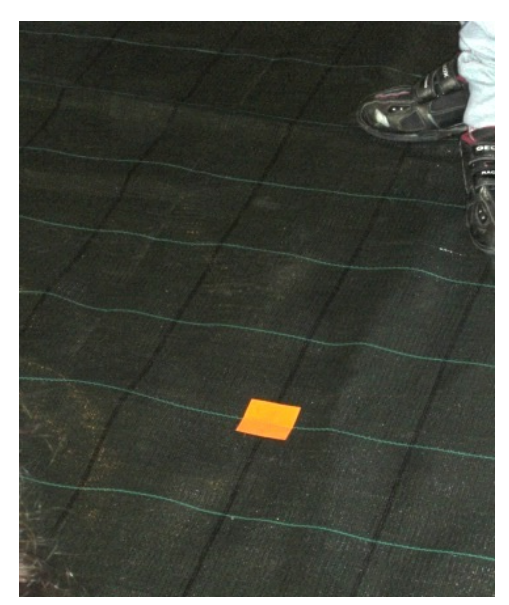

Fig 8 The squared carpet with green and black lines

First, the pupils have identified two different colored lines and described them in terms of horizontal and vertical lines. They were using their spatial knowledge, which is normal because they do not yet know the geometrical relationships between lines. Some pupils used the grid to determine the position and others to follow a path to the object (Fig. 10). The two classes worked in different ways in continuity with the discussions of phase 1. At the beginning, class A favored position and crossing 
lines, while class B favored paths and squares. This difference means that the situation assures the emergence of both ways of using the grid as model of the space, for position and path.

During phase 2 which lasted 3 lessons, class A worked alternatively with and without the carpet. During the second lesson, pupils used a graphical representation of the carpet on the blackboard in their classroom.

At the beginning of phase 2 (first lesson), lines between external landmarks of opposite sides of the carpet and crossing lines appeared in pupils' interventions. The carpet in phase 2 led pupils to identify not only lines, but also to see squares on the carpet. During the second attempt to solve the task, they considered particular lines and reference points: "there was a green line going from Giada to Giulia that pointed out that it was there [the object] and then a black [line] going from Matilde to Erika and they formed a crossing" (Badr). The identification of crossing lines on the carpet led to successful strategies, "it was easier because this time I used the references, because this time there is the grid, the green and black lines are crossed" (Matteo). Pupils referred to these lines as crossing lines, but not as knots or intersections in geometrical terms. This can be analyzed in terms of dimensional deconstruction: the pupils operate the deconstruction from 2D to $1 \mathrm{D}$ but they do not operate the deconstruction from $1 \mathrm{D}$ to $0 \mathrm{D}$ in which the position can be represented by the intersection point of two geometrical straight lines, as in this excerpt:

$\begin{array}{ll}\text { Teacher } & \text { Jennifer, the position is right. What have you looked at? } \\ \text { Jennifer } & \text { Sara and Sofia. } \\ \text { Teacher } & \text { And what have you found? } \\ \text { Jennifer } & \text { A crossing. }\end{array}$

The teacher proposed to study the task by drawing the carpet on paper and on the blackboard. In such a way, she introduced the graphical space and took into account the construction of relationships between the physical meso space and the micro space. At the same time, she aimed to help pupils to remember the tasks they previously solved. Pupils' solutions included the identification of "reference points" (material landmarks or pupils, not geometrical points) and the use of lines forming the grid. Some pupils proposed to break up the carpet into squares, which favored the emergence of a path strategy because of the possibility to count steps. The drawings of the carpet involve the meso physical space and micro graphical space, the latter as the model of the former, and can be interpreted as a representation of the path.

During the third lesson of this phase, pupils came back to the carpet in the meso space and tested the new strategy. At the end of the lesson, a new task was given: plan a path on paper (micro graphical space) in order to follow it latter on the carpet (meso space). Pupils moved around the carpet in order to make precise measurements. Some conflicts arose when pupils were not able to follow their path to the position on the carpet.

In class B, pupils worked with the carpet all along phase 2, and felt an essential need for some coding to distinguish the small squares because they are identical to each other. Some pupils proposed to color each square with a different color, which was rejected because the task would become too easy. Others proposed to number them but failed to express how to move on the carpet using numbers. The 
activity was carried out in the physical space. Only at the end of this collective work, a pupil proposed, "we can put tags with letters close to the lines on both sides and maybe we can write the pupils" names on the tags that are here and on the other side we can put the tags with numbers, by some drawings". This is similar to the strategy performed in class A and this initiated a code of the grid. As in class $\mathrm{A}$, the pupils did not talk about points and explicitly used the terms lines or squares in their discussions.

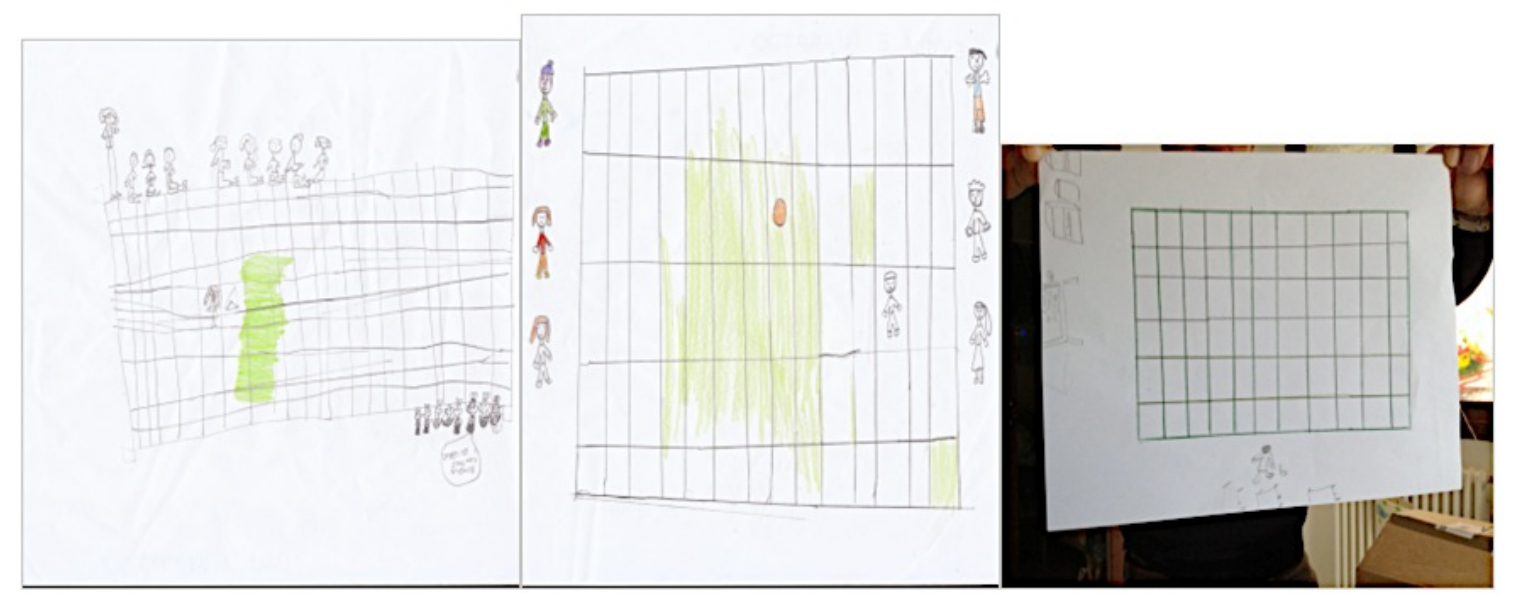

Fig. 11 Drawings show pupils' experiences with the carpet (class B)

The drawings (Fig. 11) represent different conceptualizations of the carpet with a grid corresponding to the physical situation. In the first drawing (Fig. 11, left), the pupil drew lines on the carpet in two directions with pupils around the carpet, but they were not able to distinguish the lines. In the second drawing (Fig. 11, middle), the representation of the grid and the children are consistent with the strategy, which uses pupils as landmarks. In addition, the lines are evenly spaced, although the number of lines in the drawing does not equal to the number of lines on the carpet. In the third drawing (Fig. 11, right), the focus is on the squares on the grid.

This second phase shows a gap between the spatial situation in which the grid can be used as an instrument to solve the task and the graphical space in which the grid was presented and recognized by the pupils. In particular, there is a lack of spontaneous use of graphical representations of the space as a part of a strategy that would allow pupils to replicate the path. Rather, pupils aimed at memorizing the path. Teachers' interventions took into account the construction of relationships between the two spaces and led pupils to use the micro graphical space to represent the meso space, because the situation was not strong enough to provoke it. In phase 3 of the experiment, where the Cabri e-book was introduced, the situation again required the use of a graphical representation of the spatial situation.

Pupils' discussions and drawings focused on shapes, showed dimensional deconstruction to 1D, and identified 2D figures (squares). 


\subsection{Analysis of pupils' strategies in graphical space with technology}

During phase 3, pupils worked in a graphical micro space with the task of constructing a path to reach Cabri. Pupils identified some continuity between the two spaces, like the possibility to change the point of view ("Look! Virgi we can also move the grid!"- class B). Texts written on their drawings and their remarks show that they were also aware of the specific feedback of the e-book. They contain references to strategy feedback, as a pupil remarked, "check if we write the directions..." (Figs. 12 right, 13), or evaluation feedback, "Ho vinto [I win]" or "9 coppe [cup]" (Fig. 12).

The passage to the micro space sets off the use of paper and pencil, which allowed pupils to record their trials and errors, and to improve their planning of the path. Pupils were aware of the role of diagrams in the success of their strategy (class B):

Teacher How did you solve the problem of the cloud?

Agata I have written it on a sheet.

At the end of the activity, pupils' drawings show different conceptualizations of the task and of the space in which it was performed. Table 1 shows the classification of pupils' drawings.

The three kinds of drawings are analyzed as follows:

1. Representation of the path through the reproduction of the squares on which the frog moves

In such drawings, path and coding carry similar meanings. In Fig. 12, the paths are drawn using a unit of measure (step, arrow), while in Fig. 13, only the length and the direction the path are shown.
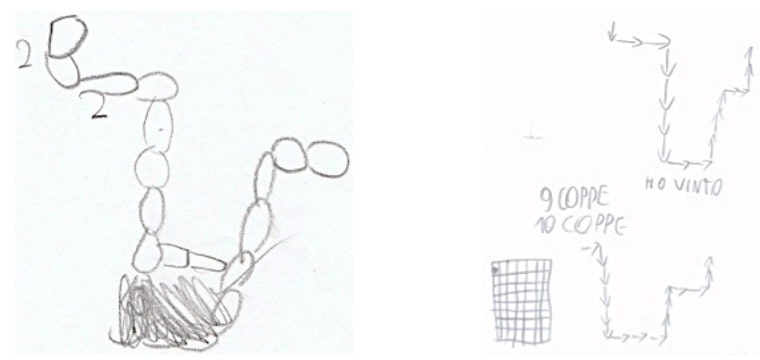

"We have seen that the squares were onwards and we have counted and searched for them. Then we did the tests when the cloud appeared. Then we watched and we checked if we wrote the right directions. If we were not right we made the path again."

Fig. 9 Drawing of the path with a unit of measure (class B on the left, class A on the right)

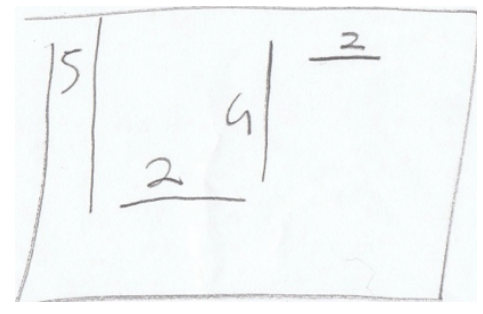

Fig 10 Drawing of the path with directions and indication of size (class B)

These three diagrams are the sign of a dimensional deconstruction, extracting some relevant 1D elements from the $2 \mathrm{D}$ situation. 
2. Representation of the whole space where the frog moves

Fig. 14 shows a pavement of 2D elements, in which there are no straight lines, no 1D elements, nor intersection points. Fig. 15 shows straight lines (1D) and the indication of obstacles.

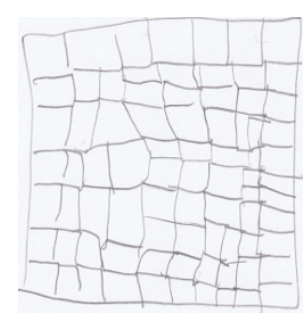

Fig. 11 Drawing of the space where the frog moves (class B)

The diagram in Fig. 15 is a very interesting one, because it is an adequate and efficient model of the situation, allowing different paths to be followed. The dimensional deconstruction occurs in the production of the diagram, where crossing straight lines are used to represent the grid. This is very different from the pavement in Fig. 14.

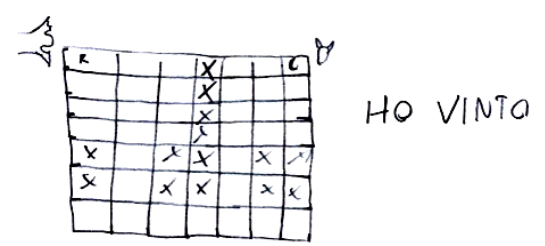

\begin{abstract}
"We have tried many times until the frog is out of the labyrinth. We have calculated all the spaces [squares]. We avoided all the traps and although there were clouds we succeeded because we had already made a representation on the sheet."
\end{abstract}

Fig. 12 Drawing of the space where the frog moves with obstacles (class A)

3. Coding of the path by arrows and numbers, without graphical representation of the path (Fig. 16) In this case, the pupils modeled the path. This model is effective because it allows them to replicate and to communicate the path.

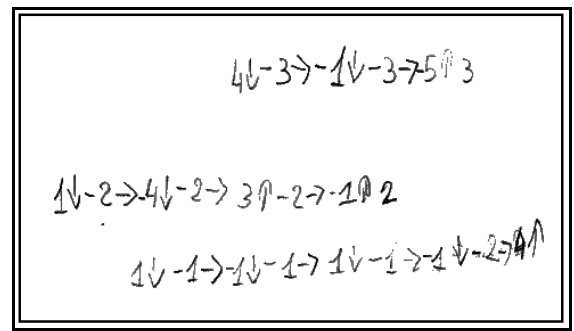

"During the counting down from 8 to 0 , we have counted the squares of the squared plane. We started to count the square from the next to the frog. We counted the steps forward, right, left, top and bottom. When there was the cloud we have tried so many times before you find the right path. We chose this representation because for us it was that we could better understand."

Fig. 13 Coding of the path (class A)

These diagrams are representations of either the space in which the frog moves or a successful path between the frog and Cabri. Some pupils' graphical representations are not complete. But they are 
linked to their strategies aiming at solving the tasks on the grid. When class B pupils have returned to the carpet after phase 3 , their productions have changed from a representation of the path to a representation of the grid and the coding of the path (Table 2).

\section{CONCLUSION}

Following Perrin-Glorian et al.'s (2013) work, we assume that the construction of geometrical knowledge is based on spatial knowledge and implies building relation- ships between the three spaces: physical, graphical and geometrical.

The "grid" experiment is a spatial problem of recording a position or a path through the use of a grid, either in the meso physical space or in the micro graphical space. It is carried out in a learning environment combining physical objects and digital technology. The aim of the experiment is to understand how the spaces are connected in pupils' strategies to solve the task.

The a priori analysis shows that the grid is a complex object, present in the three different spaces. It may be a tool to solve problems of the spatial or the geometrical field, involving spatial or geometrical knowledge. But the analysis of the phase 1 of our experiment shows pupils' use of the grid in the graphical micro space does not seem to be sufficient for constructing spatial knowledge to solve a problem in physical meso space. In fact, pupils didn't recall the grid spontaneously to solve a meso space problem, even if they had previously worked with it in micro space. Moreover, 7-year-old pupils don't have the necessary geometrical knowledge, like perpendicularity, for constructing an efficient grid, i.e. a geometrical tool to model the spatial situation.

Nevertheless, our situation supports the construction of relationships between spatial and geometrical spaces through the graphical space, a space for representing movements in the meso physical space or for coding such movements. The representation of the paths on the grid was the first link between the spaces. In these processes of representation, the dimensional deconstruction observed in pupils' diagrams shows crossing lines and reference points mapping as ways to a geometrical construction of the grid and ways to represent positions and movements on a plane. Pupils preferred the strategy of moving inside the squares of the grid and not along the lines. An analysis in terms of dimensional deconstruction brings about an explanation. The second strategy requires the conceptualization of a position as a point (0D, intersection of two $1 \mathrm{D}$ objects) while the first strategy calls for a "dot", a 2D object, avoiding dimensional deconstruction.

The use of digital technologies such as Cabri Elem e-books can support the construction of connections between spatial and geometrical fields. Indeed, it allows pupils to the use the graphical space by the play of didactical variables and it offers feedback for validation.

We use dimensional deconstruction to analyze the problem solving process in the geometrical field and geometrical reasoning. Pupils achieved 2D to 1D dimensional deconstructions when they used quadrilaterals and lines to construct a grid. But they didn't do it systematically. If they refer to geometrical properties linking the squares or the intersecting straight lines, it reveals a form of geometrical reasoning. Moreover, geometry is not the only field of knowledge that provides models to solve a spatial problem. Some pupils' diagrams question the idea that geometry would be the privileged field to model spatial situations. 
Our results contribute to the study of connections between spatial and geometrical fields. But they require further analysis of how learning situations with manipulatives in physical space and digital technology help pupils to create such connections.

\section{REFERENCES}

Balacheff, N. (2013). cKф, a model to reason on learners' conceptions. In M. V. Martinez \& A. Castro Superfine (Eds.), PME-NA Psychology of Mathematics Education North America Chapter (pp. 215). Chicago IL, USA.

Bartolini Bussi, M., \& Baccaglini-Franck, A. (2015). Geometry in early years: sowing seeds for a mathematical definition of squares and rectangles. ZDM Mathematics Education, 47(3) (this issue). doi:10.1007/s11858-014-0636-5.

Berthelot, R., \& Salin, M.-H. (1993). L'enseignement de la géométrie à l'école primaire. Grand N, $53,39-56$.

Bryant, P. (2009). Paper 5: Understanding space and its representation in mathematics. London: Nuffield Foundation.

Clairaut, A. (1741). Élements de géométrie (Imprimerie et librairie classique de Jules Delalain). Paris.

Clements, D. H., \& Samara, J. (2009). Learning and Teaching Early Maths. The Learning Trajectories Approach. New York: Routledge.

Coob, P., Confrey, J., diSessa, A., \& Schauble, L. (2003). Design experiments in educational research. Educational Researcher, 32(1), 9-13.

Duval, R. (2005). Les conditions cognitives de l'apprentissage de la géométrie: développement de la visualisation, différenciation des raisonnements et coordination de leurs fonctionnements. Annales de didactique et de sciences cognitives, 10, 5-53.

Freundenthal, H. (1973). Mathematics as an Educational Task. Dordrecht, NL: D. Reidel.

Hershkowitz, R., Parzysz, B., \& Van Dormolen, J. (1996). Space and shape. In A. J. Bishop, K. Clements, C. Keitel, J. Kilpatrick, \& C. Laborde (Eds.), International Handbook on Mathematics Education (Vols. 1-2, Vol. 1) (pp. 161-204). Dordrecht: Kluwer Academic Publishers.

Jackiw, N., \& Sinclair, N. (2006). Dynamic geometry activity design for elementary school mathematics. In C. Hoyles, J.-B.

Lagrange, S. Le Hung, \& N. Sinclair (Eds.), ICMI Study 17

Technology Revisited (Vol. 2, pp. 236-245). Hanoi Vietnam. Kaur, H. (2015). Two aspects of young children's thinking about different types of dynamic triangles: Prototypicality and inclusion. ZDM Mathematics Education, 47(3) (this issue). doi:10.1007/

s11858-014-0658-z.

Laborde, C. (2004). Come la geometria dinamica puo rinnovare i processi di mediazone delle conoscenze matematiche nella scuola primaria. In B. D’Amore \& S. Sbaragli (Eds.), La didattica della matematica: una scienza per la scuola (pp. 19-28). Bologna. 
Laborde, C., \& Capponi, B. (1994). Cabri-géomètre constituant d'un milieu pour l'apprentissage de la notion de figure géométrique. Recherche En Didactique Des Mathématiques, 14(12), 165-210.

Laborde, C., Kynigos, C., Hollebrands, K., Sträßer, R., Gutierrez, A., \& Boero, P. (2006). Teaching and learning geometry with tech- nology. Handbook of research on the psychology of mathematics education: Past, present and future (pp. 275-304). Rotterdam: Sense Publishers.

Levenson, E., Tirosh, D., \& Tsamir, P. (2011). Preschool geometry. Theory, research, and practical perspectives. Rotterdam: Sense Publisher.

Lurçat, L. (1976). L'enfant et l'espace, le rôle du corps. Paris: PUF. Mackrell, K., Maschietto, M., \& Soury-Lavergne, S. (2013). The interaction between task design and technology design in creating tasks with Cabri Elem. In C. Margolinas (Ed.), ICMI study 22 Task design in mathematics education (pp. 81-90). Royaume-Uni: Oxford.

Maschietto, M., Mithalal, J., Richard, P., \& Swoboda, E. (2013). Introduction to the papers and posters of WG4: Geometrical thinking. In B. Ubuz, C. Haser, \& M. A. Mariotti (Eds.), CERME 8 (pp. 578-584). Ankara, Turquie: Middle East Technical University.

Maschietto, M., \& Soury-Lavergne, S. (2013). Designing a duo of material and digital artifacts: the pascaline and Cabri Elem e-books in primary school mathematics. ZDM - The International Journal on Mathematics Education, 45(7), 959-971.

Mithalal, J. (2010). Déconstruction instrumentale et déconstruction dimensionnelle dans le contexte de la géométrie dynamique tri-dimensionnelle (Thèse de doctorat). Grenoble, France: Joseph Fourier. Parzysz, B. (1988). "Knowing” vs "seeing” Problem of the plane representation of space geometry figures. Educational Studies in Mathematics, 19, 79-92.

Perrin-Glorian, M.-J., Mathe, A.-C., \& Leclercq, R. (2013). Comment peut-on penser la continuité de l'enseignement de la géométrie de 6 a 15 ans ? Repères-IREM, 90, 5-41.

Piaget, J., \& Inhelder, B. (1963). The child's conception of space. London: Routledge.

Stylianides, A. J., \& Stylianides, G. J. (2013). Seeking research-grounded solutions to problems of practice: classroom-based interventions in mathematics education. ZDM - The International Journal on Mathematics Education, 45, 333-341.

Thom, J. S., \& McGarvey, L. (2015). Living forth worlds through drawing: Children's geometric reasonings. ZDM Mathematics Education, 47(3) (this issue).

Tsamir, P., Tirosh, D., Levenson, E., Barkai, R., \& Tabach, M. (2015). Early-years teachers' concept images and concept definitions: Triangles, circles, and cylinders. ZDM Mathematics Education, 47(3) (this issue). doi:10.1007/s11858-014-0641-8.

Van Hiele, P.-M. (1986). Structure and Insight: A Theory of Mathematics Education. Orlando, FL: Academic Press.

Vergnaud, G. (2009). The theory of conceptual fields. Human Development, 52, 83-94. 